\title{
Radical Treatment of an Aggressive, Recurrent Benign Inflammatory Lesion of the Tongue: A Case Report \& Review of the literature
}

\author{
Rachelle LeBlanc, Anil Sharma, Peter Spafford, \\ Brent Wilde and Rick J aggi* \\ Department of Otolaryngology-Head \& Neck Surgery, \\ College of Medicine, University of Saskatchewan, \\ Saskatoon, Saskatchewan, Canada \\ *Corresponding author: Jaggi R, Department of \\ Otolaryngology-Head \& Neck Surgery and Facial Plastic \\ Surgery, College of Medicine, University of Saskatchewan, \\ Saskatoon, Saskatchewan, Canada
}

Received: April 05, 2017; Accepted: May 03, 2017; Published: May 10, 2017

\begin{abstract}
Background: There are limited publications on the management of large aggressive recurrent benign inflammatory lesions of the tongue. These lesions have a wide range of etiologies that must be explored. Many documented reports on eosinophilic ulcers/traumatic ulcers suggest surgical excision with recurrence rates relatively low.
\end{abstract}

Case Report: This article reports on an unusual case in a 48-year-old male patient with a large left anterolateral tongue ulcer with polypoid granulation tissue that has recurred after three attempts of partial glossectomy and injection of corticosteroids. A final left hemiglossectomy with a supraclavicular island flap reconstruction was performed and is currently being followed for ongoing management.

Conclusion: Aggressive, large benign inflammatory lesions of the tongue may benefit from radical treatment if all conservative management fails. Correct diagnosis, careful surgical planning, and patient preference of treatment should all be performed while maximizing success, quality of life, and improving tongue function.

Keywords: Case report; Traumatic ulcer; Eosinophilic ulcer; Pyogenic granuloma; Tongue; Hemiglossectomy

\section{Abbreviations}

CT: Computed Tomography; EU: Eosinophilic Ulcer; PG: Pyogenic Granuloma; TUG: Traumatic Ulcerative Granuloma

\section{Introduction}

Large tongue lesions in a non -smoker, non- drinker have a wide range of etiologies. The differential diagnoses include hyperplasias, papillomas, soft tissue tumor and malignancy, particularly squamous cell carcinoma. In the present case, histopathological investigations were essential to distinguish between a variety of benign inflammatory processes of the tongue. After initial investigations the differential diagnosis was narrowed to include Traumatic Ulcerative Granuloma (TUG), Eosinophilic Ulcer (EU) and Pyogenic Granuloma (PG).

TUGs/EUs are benign lesions that are usually self-limiting. They manifest as mucosal ulceration and inflammation that extends into underlying muscle, which shows a predominance of histiocyte-like mononuclear cells and eosinophils, in addition to nonspecific acute and chronic inflammatory cells [1]. The etiopathogenesis is unknown, however, trauma seems to play an important role in the development [2]. EUs are frequently located on the tongue but can also occur in other locations such as lips, buccal mucosa, palate, gingiva and floor of the mouth $[3,4]$.

The other lesion that should be included in the differential diagnosis is PG, a hyperactive benign inflammatory lesion that most often occurs on the lips and mucosa of the oral cavity. PG lesions are exophytic, presenting with smooth or lobulated surfaces that usually bleed [5]. They typically range in size from a few millimeters to several centimeters, rarely exceeding $2.5 \mathrm{~cm}[6,7]$. The etiology of PGs is unknown, however, predisposing factors include chronic irritation, trauma, infections and hormonal factors [8]. PGs occur in a variety of ages, however, they are most common in the second decade of life. Women are affected more often, likely due to hormonal effects [9-11]. It is important to note that these lesions may recur if not removed completely [5].

The following case report describes an aggressive recurrent large benign inflammatory lesion treated with three attempts of partial glossectomy, intralesional corticosteroid injection and a hemiglossectomy with a supraclavicular island flap reconstruction.

\section{Case Presentation}

A 48-year old male presented to the Otolaryngology- Head and Neck Surgery department with a 6-week history of a left tongue lesion described as quite painful. He had a history of recurrent abscess ulcerations, which resolved after 2 weeks. He is a non-smoker, nondrinker with no significant risk factors for any cancer. Initial physical exam showed a $1 \times 2 \mathrm{~cm}$ ulcerative lesion along the left lateral tongue, which was firm on palpation. No other lesions noted on the entire sub sites of the oral cavity. Endoscopic laryngoscopy was performed and was completely normal with no base of tongue involvement.

Computed Tomography (CT) of the neck with contrast was performed. Findings suggested an asymmetric, ill-defined round
Austin J Otolaryngol - Volume 4 Issue 1 - 2017 ISSN : 2473-0645 | www.austinpublishinggroup.com LeBlanc et al. (C) All rights are reserved
Citation: LeBlanc R, Sharma A, Spafford P, Wilde B and Jaggi R. Radical Treatment of an Aggressive, Recurrent Benign Inflammatory Lesion of the Tongue: A Case Report \& Review of the literature. Austin J Otolaryngol. 2017; 4(1): 1089 


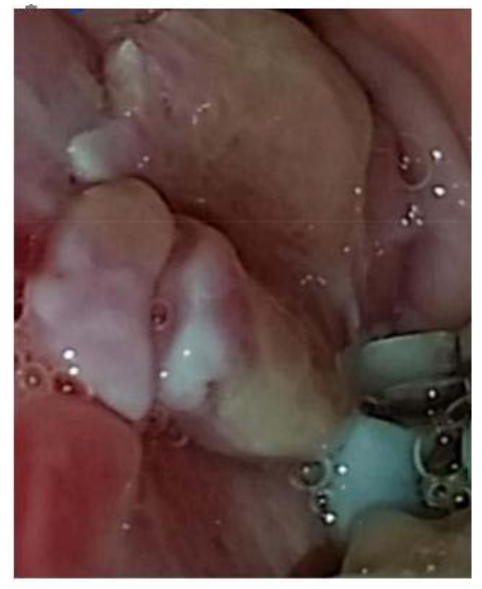

Figure 1: Day before third attempt of partial glossectomy.

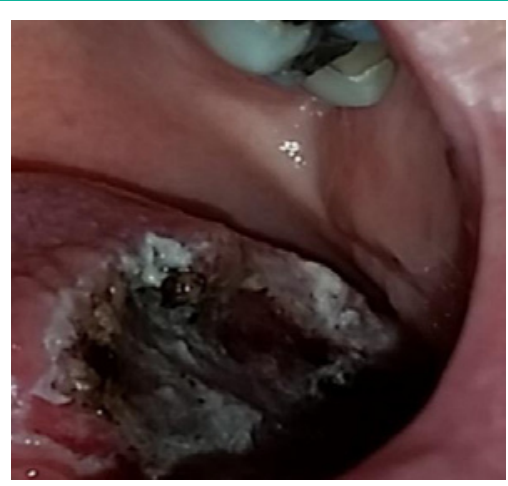

Figure 2: Post-op third attempt of partial glossectomy.

region of hypodensity measuring $22 \times 19 \mathrm{~mm}$ at the left base of tongue. There was no evidence of local extension, with no crossing over the midline. A left partial glossectomy was performed and sent to surgical pathology. The specimen consisted of squamous lined mucosa with a large central ulceration. The squamous epithelium, at the margins of the ulceration, exhibited hyperkeratosis and parakeratosis. Staining with pankeratin showed no evidence of invasive carcinoma. Immunohistochemistry and special stains did not highlight infectious organisms.

Three months later the patient returned to the department with a recurrent mass on the left lateral margin of the tongue, which was painful with intermittent bleeding. Further physical examination revealed an exophytic mass along the left lateral margin of the tongue where the previous resection had taken place. There was no active bleeding and was soft on palpation. A second resection was performed and the specimen was sent to surgical pathology. The specimen consisted of several sections of polypoid mass. The mass was lined with squamous epithelium, which in some places, exhibited marked pseudo-epitheliomatous hyperplasia. In focal areas, the squamous mucosa was ulcerated with a fibropurulent cap and underlying granulation tissue. The inflammatory infiltrate consists primarily of neutrophils with a secondary population of lymphocytes, macrophages and plasma cells. Eosinophils were also noted within the areas of ulceration and granulation tissue. At this time the findings suggested a diagnosis of an ulcer with polypoid granulation

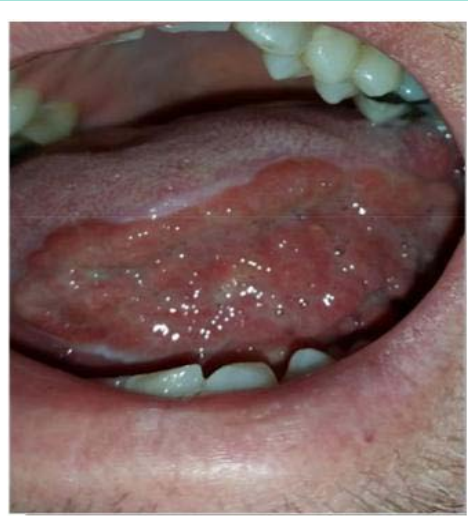

Figure 3: One month post-op third attempt of partial glossectomy.

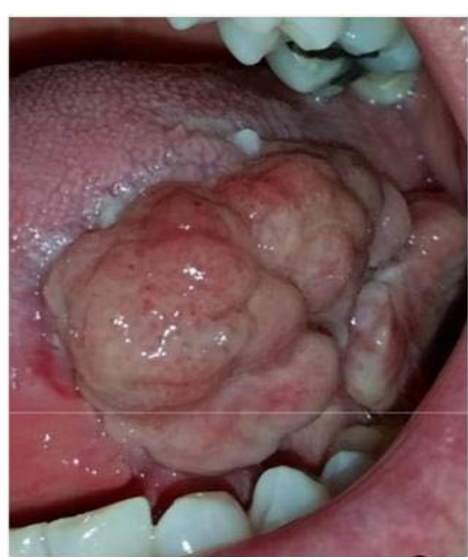

Figure 4: Two months post-op third attempt of partial glossectomy.

tissue. It did not represent a pyogenic granuloma, as it did not have characteristic lobular architecture of the lesion.

Two months later the patient was referred for a second opinion with another recurrence of the left lateral granulomatous tongue ulcer. The lesion returned with more exuberance and pain, and was bleeding. Attempt with steroid injections into the base of the granulation tissue was made and had little effect. Further examination revealed tongue deviation significantly to the left upon protrusion, implying a left hypoglossal nerve paralysis. The patient was taken back to the operating room for an excision of the left posterior floor of mouth lesion and a partial glossectomy was performed. The surgical wound was left open to heal by secondary intention (Figure 1-3). Surgical pathology revealed left tongue ulcer with extensive inflammation.

The patient returned again and was referred for a third opinion with the supervising author (RJ) two weeks after the third attempt of partial glossectomy with further recurrence. The lesion completely regrew larger than previous, involving the anterior two-thirds of the left lateral tongue (Figure 4). Endoscopic laryngoscopy showed no involvement of the base of tongue. The patient experienced significant discomfort with pain radiating to the ear, dysphagia, difficulty with speech and intermittent bleeding. The decision to perform a left hemiglossectomy with supraclavicular island flap reconstruction was made (Figure 5) and the specimen was sent to surgical pathology. Final pathology revealed ulcerated squamous mucosa with 


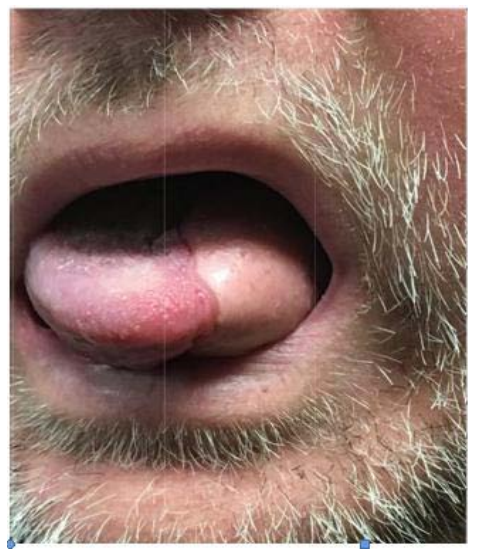

Figure 5: Post-op hemiglossectomy with supraclavicular island flap reconstruction.

granulation tissue. The squamous mucosa showed ulceration, leaving a large bed of granulation tissue. Much of the deeper inflammation was chronic in nature, rich in plasma cells and lymphocytes. There was mild atrophy and chronic inflammation involving the underlying minor salivary gland and patchy degeneration of the intrinsic skeletal

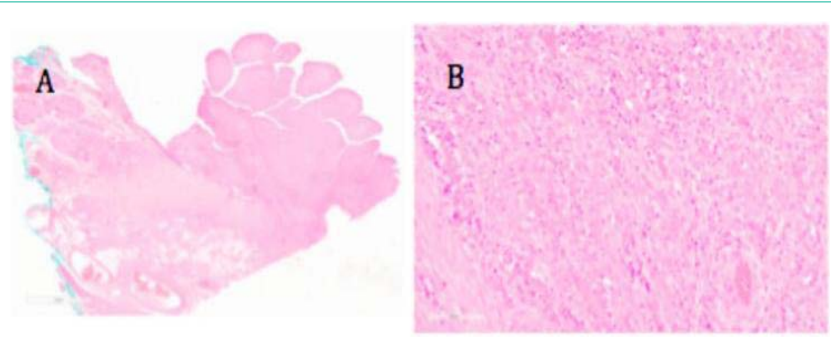

Figure 6: H\&E Staining: A) Low power field of exuberant exophytic and endophytic growth. B) Higher power showing neoangiogenesis, inflammation including eosinophils, and prominent fibroblastic proliferation.

muscle, which could be attributed to secondary alterations due to the proximity to the bed of granulation tissue. There was no evidence of dysplasia or malignancy. Neither vasculitis nor granulomatous inflammation was seen.

\section{Discussion}

There are many underlying causes of ulcerative tongue lesions (Table 1). In the present case, traumatic ulcer, eosinophilic ulcer and pyogenic granuloma are the most likely etiologies. Most benign inflammatory lesions are self-limiting if the causative agent is removed.

Table 1: Differential diagnosis of tongue ulcerations and lesions.

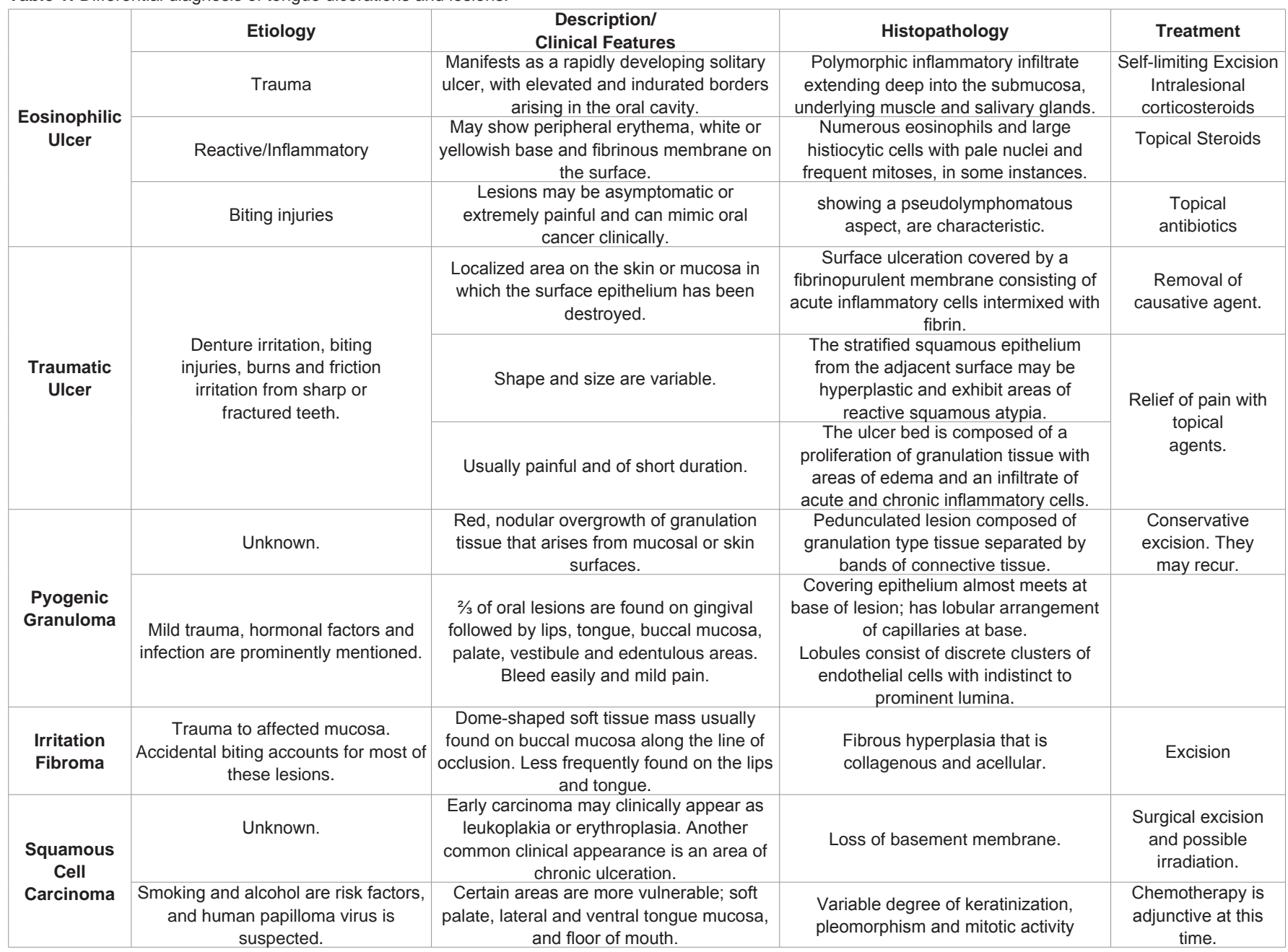


However, in the present case, it was difficult to identify the causative agent as the patient denied a history of tongue trauma, infection, smoking and alcohol use. Although the patient denied of any trauma history, the histopathology reviled abundant inflammatory cells most in keeping with a traumatic etiology. More specifically, results pointed to an eosinophilic ulcer and/or traumatic ulcer.

Eosinophilic ulcer of the oral mucosa is a benign inflammatory lesion with uncertain etiology, pathogenesis, and treatment. There have been other terms to describe it reported in the literature such as, traumatic ulcerative granuloma with stromal eosinophilia, eosinophilic granuloma of tissue and atypical histiocytic granuloma [12-14]. Popoff first described this benign inflammatory ulcer in 1956 and in 1970, the lesion was proposed as a distinct entity by Shapiro and Juhlin [15-17].

Although it is not entirely proven, Segura and Pujo suggest that EUs are traumatic in origin [12]. Though the patient in the present case denied trauma, it could be speculated that accidental bites or trauma through mastication occurred unconsciously. Vélez, et al. have suggested that the only contributing factor to the development of EUs is trauma and could potentially lead to viral or toxic agents entering the underlying tissue causing an inflammatory response [16].

Histopathology for EUs reveals poorly formed granulation tissue showing an increased number of capillaries with predominantly endothelial cells [2]. Inflammatory infiltrate of the specimen is composed of small round lymphocytes, abundant polymorphonuclear eosinophils and other inflammatory cells such as neutrophils, plasma cells and histiocytes [2]. In our case, this composition of cells was shown on histology making the diagnosis of EU/TUG most likely.

There are many different treatment modalities used for EU/TUG. Options include observation, antibiotics, systemic corticosteroids, curettage, cryosurgery and surgical excision [2]. It has been frequently reported that with surgical incision/excision there has been no further local recurrence [2]. The present case was atypical in that the lesion recurred three times before a radical approach was taken.

The present benign inflammatory lesion in this case exceeded $2.5 \mathrm{~cm}$ and was rapidly growing to an uncomfortable size. After review of literature the decision was made to take an aggressive surgical approach. Radical wide excision is usually reserved for malignant disease. In the present case, the lesion relapsed three times after conservative surgical excision and had little response to intralesional corticosteroid injection. Due to the aggressive nature of the lesion's recurrence after previous conservative surgical excisions, along with the patient's pain, nerve palsy and bleeding, a left hemiglossectomy with a supraclavicular island flap reconstruction was performed.

\section{Conclusion}

An aggressive recurrent benign inflammatory lesion was treated by three attempts of partial glossectomy and finally eradicated by a hemiglossectomy with a supraclavicular island flap reconstruction. Since the present case was aggressive in nature after failed attempts of conservative treatments, a radical approach may be a beneficial treatment option. If the lesion is large and rapidly growing, exceeding more than $2.5 \mathrm{~cm}$, radical treatment may be considered. Correct diagnosis, careful surgical planning, and patient preference of treatment should all be taken into consideration while maximizing success, quality of life, and improving tongue function.

\section{Authors' Contributions}

RL performed a chart review, data collection and drafted the manuscript. AS and PS collected the photographs and submitted data for the report. BW provided the pathology and helped draft the pathology of the manuscript. RJ conceived of the study, participated in its design and coordination and helped to draft the manuscript. All authors read and approve the final manuscript.

\section{References}

1. Almazrooa SA, John O, Sook-Bin W. Unusual large tongue ulcer. Oral Surg Oral Med Oral Pathol Oral Radiol. 2013; 116: 4-8.

2. Chandra S, Raju S, Sah K, Anand P. Traumatic ulcerative granuloma with stromal eosinophilia. Arch Iran Med. 2014; 17: 91-94.

3. Eleni G, Panagiotis S, Andreas K, Georgia A. Traumatic ulcerative granuloma with stromal eosinophilia: a lesion with alarming histo-pathologic presentation and benign clinical course. Am J Dermato-pathol. 2011; 33: 192-194.

4. Segura S, Pujol RM. Eosinophilic ulcer of the oral mucosa: a Oral Dis. 2008; 14: $287-295$.

5. Ghalayani $\mathrm{P}$, Hajisadeghi S, Babadi F. Extragingival pyogenic granuloma associated with medication: Report of an unusual case. Dental Research Journal. 2014; 11: 400-404.

6. Bouquot JE, Nikai H. Lesions of the oral cavity. In: Gnepp DR, editor. Diagnostic surgical pathology of the head and neck. $3^{\text {rd }}$ ed. Philadelphia: WB Saunders. 2002; 141-233.

7. Verma PK, Srivastava R, Baranwal HC, Chaturvedi TP, Gautam A, Singh A. Pyogenic Granuloma - Hyperplastic Lesion of the Gingiva: Case Reports. The Open Dentistry Journal. 2012; 6: 153-156.

8. Pitarch G, Perez-Ferriols A, Millan F. Recurrent pyogenic granuloma. Actas dermosifiliogr. 2012; 103: 536-539.

9. Neville BW, Damm DD, Allen CM, Bouquot JE. $2^{\text {nd }}$ ed. Philadelphia: WB Saunders; 2002. Oral and maxillofacial pathology. 437-495.

10. Regezi JA, Sciubba JJ, Jordan RCK. $4^{\text {th }}$ ed. Philadelphia: Oral pathology: Clinical pathologic considerations. 2003; 115-116.

11. Eversole LR. $3^{\text {rd }}$ ed. Clinical outline of oral pathology: Diagnosis and treatment. 2002; 113.

12. Segura S, Pujol RM. Eosinophilic ulcer of the oral mucosa: a Oral Dis. 2008; 14: 287-295.

13. Boffano P, Gallesio C, Campisi P, Roccia F. Traumatic ulcerative granuloma with stromal eosinophilia of the retromolar region. J Craniofac Surg. 2009; 20: 2150-2152.

14. Ficarra G, Prignano F, Romagnoli $P$. Traumatic eosinophilic granu- loma of the oral mucosa: a CD30+(Ki-1) lymphoproliferative dis- order ? Oral Oncol. 1997; 33: 375-379.

15. Elovic AE, Gallagher GT, Kabani S, Galli SJ, Weller PF. Wong chronic oral ulcers. Oral Surg Oral Med Oral Pathol Oral Radiol Endod. 1996; 81: 672681.

16. Vélez A, Alamillos FJ, Dean A, Rodas J, Acosta A. Eosinophilic ulcer of the oral mucosa: report of a recurrent case on the tongue. Clin Exp Dermatol. 1997; 22: 154-156.

17. Shapiro L, Juhlin EA. Eosinophilic ulcer of the tongue report of two cases and review of the literature. Dermatologica. 1970; 140: 242-250. 